\title{
T-USPEX - novel method for crystal structure prediction at finite temperatures
}

\author{
I.A. Kruglov ${ }^{1,2}$, A.V. Yanilkin ${ }^{1,2}$, A.R. Oganov ${ }^{3,1,2,4}$, \\ ${ }^{1}$ Dukhov Research Institute of Automatics (VNIIA), Moscow 127055, Russia \\ ${ }^{2}$ Moscow Institute of Physics and Technology, 141700, 9 Institutsky lane, Dolgoprudny, Russia \\ ${ }^{3}$ Skolkovo Institute of Science and Technology, Skolkovo Innovation Center 121205, 3 Nobel Street, Moscow, Russia \\ ${ }^{4}$ Northwestern Polytechnical University, Xi'an, 710072, China \\ ivan.kruglov@phystech.edu
}

Due to the ever-increasing demands on the materials with enhanced properties, the task of searching for them using atomistic modeling methods is becoming increasingly important. The problem of designing novel materials comes down to finding a global minimum in a very noisy landscape in a multi-dimensional space (potential energy surface). This problem can be solved using several methods, yet the USPEX [1,2,3] evolutionary algorithm proved its effectiveness. The USPEX limitations are calculation at zero temperatures and small number of atoms in the unit cell, since the calculation of the energy of the structures (and their selection) takes place within the framework of the density functional theory (DFT). Here we present a new method (T-USPEX) which is capable of finding stable structures at finite temperatures and pressures.

T-USPEX is based on the previously developed evolutionary algorithm USPEX. The main differences come from crystal structure relaxation at finite temperature and from the way of fitness function calculation (in this case - Gibbs free energy). Relaxation part is done using molecular dynamics in the NPT ensemble with pressure corrections considered. Gibbs free energy is calculated using thermodynamic integration with the corrections from thermodynamic perturbation theory. For these methods a big supercell is needed, so MTP [4] machine learning interatomic potentials are used. In this talk results for high-temperature phases of $\mathrm{Al}, \mathrm{Fe}, \mathrm{Ti}, \mathrm{U}$ and $\mathrm{MgSiO}_{3}$ will be presented.

[1] C. W. Glass, A. R. Oganov, and N. Hansen, Comput. Phys. Commun. 175, 713 (2006).

[2] A. R. Oganov, A. O. Lyakhov, and M. Valle, Acc. Chem. Res. 44, 227 (2011)

[3] A. O. Lyakhov, A. R. Oganov, H. T. Stokes, and Q. Zhu, Comput. Phys. Commun. 184, 1172 (2013).

[4] A. V. Shapeev, Multiscale Model. Simul. 14, 1153 (2016)

This work was supported by RFBR foundation № 19-73-00237. 